\title{
DETECTABILITY OF GRAVITATIONAL WAVE BURSTS FROM A CLASS OF NEUTRON STAR STARQUAKE GRB MODELS
}

\author{
J.E.Horvath \\ Instituto Astronômico e Geofísico \\ Universidade de São Paulo \\ Av. M. Stéfano 4200 - Agua Funda \\ (04301-904) São Paulo - SP - Brasil
}

\begin{abstract}
A large class of gamma-ray burst (GRB) models (overwhelming until recently) involve the release of energy in a neutron star quake. Even though the extreme isotropy of the GRB sky established by the BATSE experiment has now shifted the interest to cosmological models, the former starquake scenarios are still attractive and may naturally produce a gravitational wave burst which carries most of the released energy. We discuss the prospects for detection of these high-frequency bursts by the forthcoming interferometers and spheroidal antennas, emphasizing the most recent results on the distribution and nature of the GRB sources. We find that, even if the overall picture is correct, the positive detection of GRB-associated gravitational wave bursts is unlikely and therefore these events will not be a prime target for the detectors.
\end{abstract}

To appear in Int.J.Mod.Phys.D 


\section{Introduction}

More than 20 years after their discovery ${ }^{1}, \gamma$-ray bursts (hereafter GRB) continue to puzzle the astrophysics community. In spite of the availability of an expanded data set and an intense activity on theoretical modeling ${ }^{2}$, there is no general consensus about the source/sources of these events. A main ingredient for this confusing situation is the lack of firmly established counterparts at any other wavelenght, which leaves the distance scale totally undetermined for an observed highly isotropic (but inhomogeneous) distribution ${ }^{3}$. In fact, the distance scale has been postulated to be as short as $10^{4} \mathrm{AU}$ (Oort cloud scale) and as long as several Gpc (cosmological scale). At first glance it seems that the evidence points more strongly towards a cosmological origin of the bursts since in that case isotropy is a naturally expected feature. However, prior to the launch of BATSE experiment onboard the Compton Observatory ${ }^{3}$, several lines of reasoning leaded to the widespread belief that galactic neutron stars (hereafter NS) were the sources of the bursts. In fact, some kind of violent disturbance in a NS continues to be an attractive model for the events although, generally speaking, the typical distance to a burster had to be increased in order to satisfy the isotropy constraints, giving rise to the so-called "extended halo/corona" distributions.

Several lines of attack are being pursued to solve this modern version of galactic vs. extragalactic controversy. They include searches for "cosmologically stretched" bursts ${ }^{4}$, searches for repeating sources ${ }^{5,6}$ and a multi-wavelenght monitoring of the error boxes in real time ${ }^{7}$. The purpose of this work is to discuss the prospects for detecting a few bursts/yr at the forthcoming LIGO-type interferometers ${ }^{8,9}$ and spheroidal resonant antennas ${ }^{10}$. Even though it is not unlikely that important new evidence to solve the mistery becomes available prior to the implementation of these facilities in more "conventional" wavelenghts rather than gravitational waves $(\mathrm{GW})$, the significance of a positive detection from them would be such an unique opportunity to learn about NS structure and GW themselves that its importance can not be overstated. 


\section{NS models of GRBs}

In its most popular and widespread version, a NS model of GRB needs the sudden release of a substantial amount of stored energy, generally associated with the cracking of a strained solid crust. The propagation of waves then shake the frozen field lines that then radiate energetic photons. Possible scenarios have been addressed in Refs.11 and 12, see also Ref.13 for a recent review. As shown in Ref.14, even a detailed treatment of the involved physics does not dissipate several uncertainties inherent to this model. Calculations indicate that up to $\sim 10^{44} \mathrm{erg}$ may be stored as elastic energy in the crustal lattice. The energy released per quake $\Delta E$ producing a GRB can be estimated as ${ }^{14}$

$$
\Delta E=10^{38} \eta^{-1}\left(\frac{F}{10^{-6} \mathrm{erg} \mathrm{cm}^{-2}}\right)\left(\frac{r}{1 \mathrm{kpc}}\right)^{2} \operatorname{erg}=\frac{\Delta E_{\gamma}}{\eta}
$$

where $\eta$ is the (unknown) efficiency of the conversion of the energy into $\gamma$ rays which will be useful to parametrize our discussion, and $F$ is a typical burst fluence for a source located at a distance $r$.

After the quake shear and compression waves will propagate through the star. As discussed in Ref.14, a force-free configuration like the quaking NS will partition the energy as the inverse of the sixth-power of the ratio of the shear and longitudinal sound speeds. This is analogous to the Earth case ${ }^{15}$ and results from the fact that quadrupoles are the lowest modes for the force-free problem. The typical frequency at which quadupolar oscillations will produce GW that damp out this motion depends on the exact composition of the matter at densities above the nuclear saturation one. Numerical computation of those frequencies have been performed by Thorne and coworkers ${ }^{16}$ and more recently by Lindblom and Detweiler ${ }^{17}$ for a complete set of equations of state. We have chosen below to scale the estimates to the values of a $1.43 M_{\odot}$ Bethe-Johnson I NS model (see Ref.17 and references therein), having a quadrupole frequency $f_{c} \simeq 2 \mathrm{kHz}$. The frequencies arising from other choices of the equation of state differ somewhat from this value (for the given mass) only if matter is substantially stiffer or softer than the former, and the corresponding GW strenghts can be easily found if necessary. We have made no attempt to compute the actual GW waveform to be expected since this is likely to be plagued by the same uncertainties affecting the generation of GRB (see Ref.14 for a through discussion of these issues). For our purposes it is sufficient to adopt the expression of the characteristic amplitude of the waves $h_{c}$ given in Ref.18.

$$
h_{c}=2.7 \times 10^{-17}\left(\frac{\Delta E_{G W}}{M_{\odot} c^{2}}\right)^{1 / 2}\left(\frac{1 k H z}{f_{c}}\right)^{1 / 2}\left(\frac{10 k p c}{r}\right)
$$


which is given in terms of the characteristic frequency $f_{c}$ and the energy put in gravitational waves $\Delta E_{G W}$ with the corresponding distance factor $\propto 1 / r$ arising from the quadrupolar character of the emission.

We turn now to a brief characterization of the detectors, addressing GW interferometers first. As discussed in Refs. 19 and 20 the important quantity that should be calculated for the detection (besides the value of $h_{c}$ ) is the signal-to-noise ratio. For a LIGO-type broad-band interferometer the latter reads

$$
\frac{S}{N}=\frac{h_{c}}{h_{n}\left(f_{c}\right)}
$$

for an assumed optimal filtering. Here $h_{n}\left(f_{c}\right)$ is the characteristic detector noise amplitude evaluated at $f_{c}$. As shown in Ref.18, the expected sensitivities of the advanced generation to bursts is limited by photon shot-noise in the high-frequency region where $h_{n}\left(f_{c}\right) \propto f_{c}$. Inserting numbers and using eq.(3) we obtain

$$
\frac{S}{N}=10^{5}\left(\frac{\Delta E_{G W}}{M_{\odot} c^{2}}\right)^{1 / 2}\left(\frac{1 k H z}{f_{c}}\right)^{3 / 2}\left(\frac{10 k p c}{r}\right)
$$

or better, depending on several possible technological improvements under study that may increase the overall coefficient.

Up to this point we have made no use of the quaking NS hypothesis but merely restated known results with an appropiate scaling. We specialize now for the case of NS quakes by identifying $\Delta E_{G W} \leq \Delta E=\Delta E_{\gamma} / \eta$ and write eqs.(2) and (4) as

$$
\begin{gathered}
h_{c}=2.7 \times 10^{-17} \eta^{-1 / 2}\left(\frac{\Delta E_{\gamma}}{M_{\odot} c^{2}}\right)^{1 / 2}\left(\frac{1 k H z}{f_{c}}\right)^{1 / 2}\left(\frac{10 k p c}{r}\right) \\
\frac{S}{N}=10^{5} \eta^{-1 / 2}\left(\frac{\Delta E_{\gamma}}{M_{\odot} c^{2}}\right)^{1 / 2}\left(\frac{1 k H z}{f_{c}}\right)^{3 / 2}\left(\frac{10 k p c}{r}\right) .
\end{gathered}
$$

Since $\eta$ is a small number (of the order of a few percent at most), most of the energy comes out in GW for an oscillating star ${ }^{11}$. In what follows, we shall consider the sensitivity of an advanced LIGO detector ${ }^{19}$ and consider $S / N>2$ as a reasonable criterion for detection ${ }^{20}$. Optimal filtering and orientation of the interferometer(s) are also assumed as done in previous works. Before addressing the astrophysical setting of the sources we should remark that the GRB detection offer in principle important advantages when compared to other possible GW bursts. Since the gammas would act as an electromagnetic counterpart of the GW signal, they could be identified below the sensitivity threshold calculated for a random distribution of the bursts ${ }^{19}$ (i.e. those not associated with a GRB error box). This 
results in an additional factor $\sim 3$ already taken into account in the above expressions for $h_{c}$ and $S / N$. The position of the GRB would be a powerful check for the independently determined position of the GW by two or more detectors, with the corresponding gain of physical information about the sorces and the waveform itself.

An alternative and very promising complementary technique to investigate the high-

frequency range is the recent proposal of several spheroidal resonant antennas ${ }^{10,21}$. The goals of this " $4^{t h}$ " generation of detectors cooled to $T \leq 0.1 \mathrm{~K}$ are to reach high sensitivities of $h_{c} \sim 10^{-21}$ or better in a short construction timescale. In particular, it is expected that a truncated icosahedron design can be not only $\sim 50$ times more sensitive than a bar antenna with the same noise temperature, but also that the direction and polarization of the wave can be measured at once on each of these systems. According to the calculations of Ref.22 an $1.3 \mathrm{~m}$ diameter buckyball of $A l$ having a central frequency of $2 \mathrm{kHz}$ and a bandwidth $\Delta f \sim 100 \mathrm{~Hz}$ would have a strain noise spectrum one orderof-magnitude lower than an optimally oriented first-generation LIGO-type interferometer and comparable to the advanced generation estimates (other materials and geometries may be even more useful and are under study). This sensitivity will result in a similar $S / N$ ratio than the eq.(6) one, but given the potential operability in $\sim 3 \mathrm{yr}$ because of fewer foreseen technological problems ${ }^{22,23}$ they may become available faster than the long-shot advanced LIGOs. In the remaining of this work we shall assume that either technology will be finally able to observe the possible GRB-associated emission.

\section{Detectability of the GW from GRB burst sources}

As stated in the Introduction, the confirmed isotropy of the GRB sky diminished the confidence the researchers had prior to BATSE launch about the correctness of the NS picture. However, for a variety of reasons NS should still be considered as likely sources. In fact, many variants of the latter model have been constructed ${ }^{24}$ and it has been claimed that the data is indeed consistent with a repeating population of galactic $\mathrm{NS}^{25}$. Previous analysis of the possible GW events produced by vibrating NS can be found in Refs.26 and 27 , although these works did not address recent GRBs models but rather concentrated on the general features of the quakes. We shall discuss the GW detection of several subclasses of GRBs following the most recent advances in the understanding of the latter.

a) "Classical" GRB : The availability of BATSE data on the dipole and quadrupole moments of the classical GRB distribution has challenged the view of model builders. Both quantities $\mathbf{D}=\left\langle\cos \theta>\right.$ and $\mathbf{Q}=<\sin ^{2} b>-1 / 3$ are amazingly close to zero and since 
the brightness distribution requires a decrease of the number of sources with distance ${ }^{3}$, it is difficult to model them as a known component of the galactic disk which requires the typical distance to the sources $d$ to be less than the scale height of the disk $Z_{D}$ to account for the data. However, there have been claims that this is the case and that GRB are associated with the galactic arms at $\sim 1 k p c$ distance scale ${ }^{25}$. In such a case, there would be no need of extended halo/extragalactic sources to explain the events. Given the typical fluence $F=10^{-6} \mathrm{erg} \mathrm{cm}^{-2}$ of a GRB we get, according to eq.(1) $\Delta E_{G W} \simeq 10^{38} \eta^{-1} \mathrm{erg}$ and therefore $S / N=3 \times 10^{-3} \eta^{-1 / 2}$. Thus, a burst would be detectable (i.e. $S / N>2$ ) if $\eta \leq 3 \times 10^{-6}$. In other words the actual efficiency of the conversion to gammas must be very low for the associated GW to be detected, so low that in the latter case each event must release at least $\Delta E \geq 3 \times 10^{43} \mathrm{erg}$ or about $10 \%$ of the total elastic energy stored in the crust. This is a very severe requirement since, if a local galactic population alone is invoked, the observed GRB rate of $1 /$ day calls for at least $10^{5}$ bursts/NS over a Hubble lifetime of the object. It is apparent that if this is the case, the quantity $\Delta E$ must be much lower (higher $\eta$ ) and therefore the GRB events will not be seen by the forthcoming detectors.

There is, however, another popular modeling of the sources involving NS in which an extended halo/corona is the main responsible for the isotropy without excluding a disk NS contribution. The models of Higdon and Lingenfelter ${ }^{28}$ and Smith and Lamb ${ }^{29}$ are examples of dual populations. Li et al. ${ }^{30}$ propose that high-velocity NS populate the halo and produce the events. Hakkila et al. ${ }^{31}$ and Smith $^{32}$ have shown that a significant fraction of the sources (up to $30 \%$ ) can be in the disk, so that in these cases the energy problem discussed above may be avoided since the disk NS would not be required to reproduce the whole distribution. But even if this is the case it is not automatically guaranteed that the GRB from the disk can produce GW signals at an intersting rate $\sim 10$ events/yr. Let us assume that $\eta$ is much higher, say $\geq 10^{-3}$ as seems reasonable. If so $\Delta E \sim 10^{41} \mathrm{erg}$ and with the same criterion given above for a positive detection we obtain that the distance to the source can not be larger than $r_{m} \simeq 40-50 p c$. The closest NS out of the $10^{9}$ present in the galaxy is likely to be $\sim 10 p c$ away $^{33}$ and therefore there are at least 100 potential sources in a sphere of radius $r_{m}$. Thus, since we are sampling $f e w \times 10^{6}$ out to $\sim k p c$ scale and in these composite models the latter can produce $\sim 10 \%$ of the annual events, the probability of observing a burst closer than $50 \mathrm{pc}$ (so that it can be also detected in GW) is $P \sim 10^{-3}$. Even if the Quashnock-Lamb results hold and the whole disk NS population $^{34}$ adds up to produce the events, $P$ increases to a meager $2 \%$. Needless to say, these are not very encouraging numbers.

The best prospects for GW detection arise if the very intense events like GRB 910601 
having $F=5 \times 10^{-5}$ are simply the closest to the Earth from an extended halo/corona distribution. From eq.(1) we see that its distance should be about $0.15 R$, ( where $R$ is the typical distance to a souce $\sim 10-20 \mathrm{kpc}$ in these models). In such a case the associated GW may be detectable if $\eta<10^{-3}$ which is small but perhaps not unreasonable.

\section{b) "Soft" Gamma Repeaters}

According to most researchers, the so-called soft-gamma repeaters (hereafter SGR) represent a subclass of $\gamma$ transients differing from their "classical" cousins because they present

i) Stochastic recurrence patterns and short repetition times

ii) Average duration peaked at $\sim 1 s$.

iii) Constant spectral shapes with maximum output at $E \sim 30 \mathrm{keV}$.

iv) Lack of substantial spectral evolution.

v) Rapid rise and decay timescales, unresolved in most cases.

Only three repeating sources heve been identified as such, notably SGR 0526-66 coincident with the position of the celebrated 1979 March 5 superburst $^{35}$. We discuss this association first, having in mind the widespread (but not necessarily correct) picture that superbursts are a manifestation of an internal phenomenon (e.g. phase transitions ${ }^{36}$ ) that triggers and active period of SGR of the source.

\section{* 1979 March 5 event (SGR 0526-66)}

This is the most celebrated GRB event recorded so far, although it is not considered itself as a part of the SGR class. Among its unique features, the exceedingly large fluence allowed a detection by 12 instruments, making possible a quite precise localization. Its association with the LMC supernova remnant $\mathrm{N} 49^{35}$ was subsequently debated until recently (see below), but there is now firm evidence for a LMC origin at $D=50 \mathrm{kpc}$ strenghtened by the identification with supernova remnants of the remaining two sources. The position is also consistent with the source $S G R 0526$ - 66, very suggestive of a scenario in which $G R B 050379$ triggered an "active" period of the former, identified with a young NS. Assuming an isotropic emission of $G R B 050379$, the detected fluence implies $\Delta E_{\gamma} \geq 10^{44.8} \mathrm{erg}$. Our estimation for GW is then $S / N=0.132 \eta^{-1 / 2}$; suggesting detectability of the burst if $\eta \leq 5 \times 10^{-3}$. Even though there is a considerable uncertainty on the nature of the event and the precise form of the $\gamma$ flash generation, the constraint on $\eta$ seems not too strong. Furthermore, Ramaty et al. ${ }^{36}$ have demonstrated that the temporal history of the event is compatible with GW damping of a vibrating NS but the elastic crust energy considerations do not apply since the free energy source is likely to be 
associated with core phase transitions. However, it should be remembered that the event remains unique in more than 20 years of GRB observations and thus the frequency at which the galaxy and local extragalactic neighbours produce potentially detectable events disfavors them as promising targets.

* SGR 1806-20, SGR 1900+14 and SGR 1806-20

These three identified sources of SGR have attracted lots of attention since the identification with supernovae remnants ${ }^{37,38}$ and the confirmation by the GINGA satellite team $^{39}$. It now appears that the distance scale is rather well established $(D \sim 15 \mathrm{kpc}$ for SGR 1806 - 20 in SNR G10.0-0.3 . and $D \sim 50 k p c$ for $S G R 0526$ - 66 in N49) and the hypothesis of a quaking NS leftover from the explosions seems reasonable ${ }^{40}$, even though alternative explanations invoking abnormally high magnetic fields have been put forward ${ }^{41}$ so that vibration of the star is not compellingly involved. Adopting $15 \mathrm{kpc}$ as the actual distance we get $\Delta E \simeq 10^{41} \eta^{-1} \mathrm{erg}$ for the strongest bursts of $S G R 1806-20$ having $F \sim 3 \times 10^{-5} \mathrm{erg} \mathrm{cm}^{-2}$. Our estimate is then $S / N=6 \times 10^{-3} \eta^{-1 / 2}$ and the GW bursts possibly associated with the sources will be detectable if $\eta \leq 8 \times 10^{-6}$. The total energy that must be radiated per event is well above the limiting value that can be stored in the crust (and this figure must yet be multiplied by the total number of events for a given source). Even without considering the emission of GW (i.e. setting $\eta \simeq 1$ ) the total energy radiated in GRB happens to be greater than the elastic crust value and the latter has to be replenished to match the energetic requirements of the observed active periods. These numbers suggest that although NS are strong candidates for SGR origin, it is unlikely that quakes can provide an explanation for them, and thus GW bursts need not to arise at any intensity level after all.

\section{Conclusions}

We have discussed in this work the detectability of GW bursts possibly associated with a definite model of GRB generation, namely the quakes of NS. This model has been paradigmatic until recent recent results announced by the BATSE team established clearly that, even though a fraction of them may originate in this fashion, it is unlikely that the model can provide a full explanation for the data by itself. This fact shifted the interest to cosmological alternatives, but the debate is not likely to end soon. We have tried to express our estimates as closely related as possible to these new GRB data. Our conclusion is that "classical" GRB, assumed to be produced by NS quakes and allowed to be up to $30 \%$ of the whole distribution may be seen in future GW detectors if one of the burst sources lies within 
$50 p c$, but the chance probability of such an event is $\sim 10^{-3}(0.02$ in the extreme case that all them are galactic as advocated by Quashnock and Lamb ${ }^{25}$ ). There may be a reasonable prospect for GW detection if most of the classical bursts arise form extended halo/corona distributions and the very intense ones are the closest $\sim$ few $k p c$ away. Events like the famous 1979 March 5 would be observable by LIGO-type interferometers and buckyball arrays, but they seem to be too rare to produce a significative rate. Finally, we found that SGR, now known to be associated with young NS are not likely to be detected unless our knowledge of the elastic properties of dense matter is grossly wrong and those compact objects are more exotic than we think ${ }^{42}$. A similar analysis of GRB-GW coincidence for the popular NS-NS inspiraling cosmological sources has been made by Nicholson and Schutz ${ }^{43}$. These type of works represent the first attempts towards a "multi-wavelenght" study of high-energy phenomena that will be greatly stimulated by GW detectors operation in the near future.

\section{Acknowledgements}

We acknowledge Dr. O.D. Aguiar for his interest in this problem and valuable information and advice. The financial support of the Conselho Nacional de Desenvolvimento Científico e Tecnológico $(\mathrm{CNPq})$, Brazil is also acknowledged. 


\section{References}

1. R.W.Klebesadel, I.B.Strong and R.A.Olson, Astrophys.J.Lett. 182, L85 (1973).

2. R.Nemiroff, in Proceedings of the $2^{\text {nd }}$ Huntsville Workshop on Gamma-Ray Bursts, AIP Conf. Proc. 307, G.Fishman,J.Branierd and K.Hurley (Eds.), 730 (1994).

3. C.Meegan et al., Nature 355, 143 (1992).

4. S.P.Davies et al., in Ref.2, 182.

5. J.M.Quashnock and D.Q.Lamb, Mon.Not.R.A.S. 265, L69 (1993).

6. V.C.Wang and R.E.Lingenfelter, in Ref.2, 160.

7. S.D.Barthelmy et al., in Ref.2, 643.

8. A.Giazzoto, Phys. Rep. 182, 367 (1989).

9. R.Vogt, talk given at the $12^{\text {th }}$ International Conference on General Relativity and Gravitation (GR12).

10. See, for example, O.D.Aguiar et al., in Proc. of the $13^{\text {th }}$ International Conference on General Relativity and Gravitation (GR13), W.Lamperti and O.E.Ortiz (Eds.), 455 (1993).

11. A.I.Tsygan, Astron.Astrophys. 44, 21 (1975) ; A.C.Fabian, V.Icke and J.E.Pringle, Astrophys. Space Sci. 42, 77 (1976).

12. F.Pacini and M.Ruderman, Nature 251, 399 (1974) ; M.Ruderman, Astrophys. J. 382, 587 (1991).

13. E.P.Liang, in Proceedings of the GRO Science Workshop, N.Johnson (Ed.), 397 (1989).

14. O.Blaes, R.D.Blandford and P.Goldreich, Astrophys.J. 343, 839 (1989).

15. T.Kasawara, Earthquake Mechanics, (J.Wiley \& Sons, NY 1981).

16. K.S.Thorne and A.Campolattaro, Astrophys.J. 149, 591 (1967) ; R.Price and K.S.Thorne, Astrophys.J. 155, 163 (1969) ; K.S.Thorne and A.Campolattaro, Astrophys.J. 159, 847 (1970). 
17. L.Lindblom and S.Detweiler, Astrophys.J.Supp. 53, 73 (1983).

18. K.S.Thorne, in 300 Years of Gravitation, S.Hawking and W.Israel (Eds.), 330 (Cambridge University Press, London 1987).

19. A.Abramovici et al., Science 256, 325 (1992).

20. D.Kennefick, Phys. Rev. D 50, 3587 (1994).

21. W.O.Hamilton and W.W.Johnson, Proposal for a Gravitational Wave Observatory, proposal to the National Science Foundation (1990) ; O.D. Aguiar et al., communication to the Proceedings of the Cryogenic Gravitational Wave Antenaae Progress Workshop, Legnaro, Italy (1993).

22. W.W.Johnson and S.M.Merkowitz, Phys.Rev.Lett. 70, 2367 (1993).

23. O.D.Aguiar, private communication.

24. D.H.Hartmann, in Ref.2, 562.

25. J.M.Quashnock and D.Q.Lamb, Mon.Not.R.A.S. 265, L45 (1993).

26. K.S.Thorne, in Theoretical Principles in Astrophysics and Relativity N.R.Lebovitz, W.H.Reid and P.O.Vandervoort (Eds.) (University of Chicago Press, Chicago 1978).

27. P.Haensel, J.L.Zdunik and R.Schaeffer, Astron.Astrophys. 160, 251 (1986).

28. R.E.Lingenfelter and J.C.Higdon, Nature 356, 132 (1992) ; J.C.Higdon and R.E.Lingenfelter, in Ref.2, 586.

29. I.A.Smith and D.Q.Lamb, Astrophys.J. 410, L23 (1993).

30. H.Li, R.Duncan and C.Thompson, in Ref.2, 600.

31. J.Hakkila et al., in Ref.2, 59.

32. I.A.Smith, in Ref.2, 610.

33. D.Eichler, in Ref.2, 54. 
34. R.N.Manchester and J.H.Taylor, Pulsars (Freeman, San Francisco 1977).

35. T.L.Cline et al., Astrophys.J. 255, L45 (1982).

36. R.Ramaty et al., Nature 287, 122 (1980).

37. D.A.Frail and S.R.Kulkarni, in Ref.2, 486.

38. S.R.Kulkarni and D.A.Frail, Nature 365, 33 (1993).

39. T.Murkami et al., in Ref.2, 489.

40. J.P.Norris et al., in Ref. 13, 479.

41. R.C.Duncan and C.Thompson, in Ref.2, 625.

42. B.Schutz, in Ref. 10, 191.

43. D.Nicholson and B.Schutz, in Ref. 10, 388. 